\title{
Estadísticas de recursos financieros públicos para la salud en México, 2001*
}

E n México, los recursos públicos disponibles para el financiamiento de la salud son escasos, y aunque la información que existe es limitada, la Secretaría de Salud se ha dado a la tarea de sistematizar el gasto público en salud, en cifras totales, desagregadas por rubro de gasto $y$ funciones del sistema.

El primer ejercicio de estimación de Cuentas Nacionales de Salud en México fue realizado por la Fundación Mexicana para la Salud, institución que integró las Cuentas Nacionales de Salud de México para el periodo 1992-1998. ${ }^{1}$

El Programa Nacional de Salud 2001-2006 establece la creación de un Sistema de Cuentas Nacionales y Estatales de Salud (Sicuentas) que proporcione información que dé seguimiento a los recursos que se invierten y consumen en la producción de servicios de salud. En este sentido, el Sicuentas genera información sobre flujos financieros a escala estatal y nacional, complementa las estadísticas del Sistema de Cuentas Nacionales del Instituto Nacional de Estadística, Geografía e Informática (INEGI) y crea la información necesaria para el análisis del gasto en salud. Del mismo modo, contribuye como un insumo en la evaluación del desempeño del sistema y coadyuva a la rendición de cuentas en lo referente al uso de recursos.

El modelo de Cuentas Nacionales de Salud se basa en un esquema de flujos financieros que refleja la captación, movilización y asignación de recursos, así como la compra y pago de servicios de atención a la salud. ${ }^{2}$ La integración de dichos flujos parte de tres categorías centrales: fuentes, agentes de financiamiento y prestadores de servicios. La clasificación de estas tres categorías se basa en la Clasificación Internacional de Cuentas de Salud, lo que permite comparaciones entre países y diferentes sistemas de salud.

La información que aquí se presenta forma parte del Boletín de Información Estadística de 2001, que a partir de este año publica por separado el componente relacionado con recursos financieros. Se trata de un esfuerzo realizado por las entidades federativas y las instituciones de seguridad social y Petróleos Mexicanos (Pemex) por presentar de manera homogénea y comparable los flujos financieros del año de referencia.

De acuerdo con las cifras compiladas por Sicuentas en $2001^{3}$ se presenta una gran variabilidad del gasto entre los estados. La situación es diferente si se analiza, ya sea en fun- ción de las fuentes de financiamiento o por rubro de gasto; asimismo, si se hace referencia a la población asegurada o no asegurada.

Adicionalmente, se analiza la distribución del gasto público en salud por tipo de población, fuente de financiamiento, rubro de gasto y entidad federativa. Dicho análisis permite conocer las diferencias que existen en el financiamiento público de la salud entre los estados, además de arrojar resultados sobre los recursos que se canalizan para atender a la población no asegurada y asegurada.

Del presupuesto público total ejercido, los recursos canalizados al Distrito Federal (DF) representan más de la quinta parte del total nacional (35 971 millones de pesos corrientes, que equivale a $23 \%$ ), mientras que en estados con un importante rezago en cuanto a mortalidad infantil y de adultos, como Puebla, Chiapas, Guerrero, Oaxaca e Hidalgo presentan una participación menor a $4 \%$ del total nacional (cuadro I).

Por otro lado, al analizar los recursos que se destinan por tipo de población, se observa que el DF se mantiene como la entidad federativa que mayores recursos recibe, tanto para población no asegurada como

\footnotetext{
* Sección preparada por la Dirección General de Información y Evaluación del Desempeño. Secretaría de Salud. México.
} 
para asegurada $(14 \%$ y $28 \%$, respectivamente). Respecto a los estados con rezago en mortalidad observamos que cuando se trata de recursos para población no asegurada, éstos ocupan las posiciones 5, 6, 7, 9 y 12 (Chiapas, Oaxaca, Puebla, Guerrero e Hidalgo, respectivamente), pero cuando se refiere a recursos para población asegurada su posición cae (cuadro I).

Al presentar los recursos públicos destinados a salud para población no asegurada por fuente de financiamiento, es decir, recursos federales para entidades federativas y municipios (ramo 33), recursos federales asignados al sector salud (ramo 12) o aportaciones estatales, podemos observar que en la participación dentro de ramo 12, el DF y el Estado de México son las entidades que mayores recursos reciben, $17 \%$ y $13 \%$ del total nacional, respectivamente, en tanto que Nayarit, Campeche, Tlaxcala, Quintana Roo, Aguascalientes, Colima y Baja California Sur son los estados que menos recursos reportan, con menos de $1 \%$ cada uno (cuadros II y III).

Al considerar los recursos estatales, tres estados representan casi $64 \%$ del total nacional (DF con 37\%, Jalisco $14 \%$ y Tabasco $13 \%$ ) mientras que las demás entidades participan con porcentajes bajos que van de $4.5 \%$ (Sonora y Guanajuato), a menos de 1\% (Oaxaca, Chiapas, Hidalgo, Guerrero, Colima, entre otros).

Es importante destacar que al hablar del presupuesto ejercido o gasto por fuente de financiamiento, para población no asegurada, en casi todos las entidades (excepto Tabasco y DF) los recursos canalizados a través del Fondo de Aportaciones para los Servicios de Salud (FASSA) representan más de $40 \%$ del gasto en cada entidad (en Colima y Baja Ca- lifornia Sur llega a ser de aproximadamente $80 \%$ ) (cuadro IV).

La distribución por rubro de gasto (personal, operación e infraestructura) presenta gran similitud entre los recursos destinados a población no asegurada y asegurada, pues el rubro que más recursos capta es el de personal, $61 \%$ para población no asegurada y $71 \%$ en población asegurada, mientras que para operación e infraestructura disminuye a $36 \%$ y $3 \%$ en población no asegurada y $27 \%$ y $1.5 \%$ en población asegurada (cuadros V y VI).

Se identifican diferencias en cuanto al destino de las aportaciones estatales; mientras el promedio nacional para personal, operación e infraestructura es de $61 \%, 25 \%$ y $14 \%$, respectivamente, las entidades presentan una gran variabilidad en cuanto a la proporción del presupuesto que destinan a cada uno de estos rubros (cuadro VII).

Respecto al gasto destinado a programas de combate a la pobreza Programa de Ampliación de Cobertura (PAC), Programa de Educación, Salud y Alimentación (Progresa) e IMSS-Solidaridad destaca el hecho de que los estados que mayores transferencias reciben para ello son Chiapas, Oaxaca, Veracruz, Puebla e Hidalgo, entidades caracterizadas por una gran concentración de grupos marginados, y consideradas tradicionalmente con grandes rezagos socioeconómicos, razón por la que no es sorprendente este hallazgo. En conjunto estos cinco estados representan casi $50 \%$ del gasto destinado al combate a la pobreza (cuadro VIII).

Otro criterio para evaluar las diferencias, a escala estatal, del esfuerzo y recursos canalizados al sector salud es la distribución per capita del presupuesto público en salud. Esto nos permite establecer la cantidad de dinero que en promedio corresponde a cada individuo de una población determinada.

Se observa, a escala nacional, que el promedio de gasto per capita para población no asegurada y asegurada es de 1045 y 1988 pesos, respectivamente, lo que significa que en población asegurada se gasta $90 \%$ más en cada persona (cuadro IX).

El trabajo de Sicuentas incluye la concertación de cifras de gasto en salud con diferentes dependencias (Secretaría de Hacienda y Crédito Público, Secretaría de Salud, Instituto Mexicano del Seguro Social, Instituto de Seguridad y Servicios Sociales de los Trabajadores del Estado, Pemex) y entidades federativas, lo que ha permitido homogeneizar los criterios de clasificación del gasto, tener un mejor acceso a la información pública y, de manera destacada, obtener información sobre el esfuerzo estatal para el financiamiento de la salud.

Sin embargo, para poder contar con información completa del gasto en salud en México, queda aún pendiente la incorporación del gasto privado por entidad federativa (gasto de los hogares, de empresas, de organismos no gubernamentales). Se espera incluir esta información a Sicuentas el próximo año.

\section{Referencias}

I. Cruz C,Alvarez F, Frenk J. Las cuentas nacionales de salud y el financiamiento de los servicios. México, DF: Funsalud, 1994.

2. Organización de Cooperación y Desarrollo Económico. Sistema de Cuentas Nacionales de Salud. OECD, 200

3. http:// www.salud.gob.mx/. 


\section{Cuadro I \\ Presupuesto público ejercido por entidad federativa. México, 200 I (MILlones DE PESOS CORRIENTES)}

\begin{tabular}{|c|c|c|c|c|c|c|}
\hline Entidad federativa & Población no asegurada & $\%$ & Población asegurada & $\%$ & Presupuesto público total ejercido & $\%$ \\
\hline Nacional & 52281.8 & 100.0 & 103803.3 & 100.0 & 156085.1 & 100.0 \\
\hline Aguascalientes & 623.3 & 1.2 & I 130.1 & I.I & I 753.4 & 1.1 \\
\hline Baja California & 805.6 & 1.5 & 3192.8 & 3.1 & 3998.4 & 2.6 \\
\hline Baja California Sur & 359.9 & 0.7 & 884.0 & 0.9 & I 243.9 & 0.8 \\
\hline Campeche & 615.3 & 1.2 & 839.8 & 0.8 & I 455. I & 0.9 \\
\hline Coahuila & 937.1 & 1.8 & 3662.6 & 3.5 & 4599.7 & 2.9 \\
\hline Colima & 348.8 & 0.7 & 679.4 & 0.7 & I 028.2 & 0.7 \\
\hline Chiapas & 2399.3 & 4.6 & 1306.5 & 1.3 & 3705.8 & 2.4 \\
\hline Chihuahua & 1212.4 & 2.3 & 3678.7 & 3.5 & 4891.1 & 3.1 \\
\hline Distrito Federal & 7354.1 & 14.1 & 28617.1 & 27.6 & 35971.2 & 23.0 \\
\hline Durango & 881.5 & 1.7 & I 474.8 & 1.4 & 2356.3 & 1.5 \\
\hline Guanajuato & | 831.3 & 3.5 & 3322.4 & 3.2 & 5153.7 & 3.3 \\
\hline Guerrero & 1891.9 & 3.6 & I 479.8 & 1.4 & 3371.7 & 2.2 \\
\hline Hidalgo & I 478.0 & 2.8 & I 289.1 & 1.2 & 2767.1 & 1.8 \\
\hline Jalisco & 3310.7 & 6.3 & 6545.1 & 6.3 & 9855.8 & 6.3 \\
\hline México & 5686.1 & 10.9 & 7113.5 & 6.9 & 12799.6 & 8.2 \\
\hline Michoacán & 1803.6 & 3.4 & 2113.8 & 2.0 & 3917.4 & 2.5 \\
\hline Morelos & 804.0 & 1.5 & I 385.7 & 1.3 & 2189.6 & 1.4 \\
\hline Nayarit & 588.4 & 1.1 & 941.5 & 0.9 & I 529.8 & 1.0 \\
\hline Nuevo León & I I 148.4 & 2.2 & 5926.1 & 5.7 & 7074.5 & 4.5 \\
\hline Oaxaca & 2169.8 & 4.2 & 1141.0 & 1.1 & 3310.9 & 2.1 \\
\hline Puebla & 2006.4 & 3.8 & 2922.7 & 2.8 & 4929.1 & 3.2 \\
\hline Querétaro & 712.4 & 1.4 & 1242.2 & 1.2 & I 954.6 & 1.3 \\
\hline Quintana Roo & 585.4 & 1.1 & 1030.2 & 1.0 & 1615.6 & 1.0 \\
\hline San Luis Potosí & 1140.9 & 2.2 & 1746.3 & 1.7 & 2887.2 & 1.8 \\
\hline Sinaloa & I 267.1 & 2.4 & 2627.7 & 2.5 & 3894.8 & 2.5 \\
\hline Sonora & I 299.6 & 2.5 & 2931.5 & 2.8 & 4231.0 & 2.7 \\
\hline Tabasco & 1956.4 & 3.7 & I 691.I & 1.6 & 3647.6 & 2.3 \\
\hline Tamaulipas & I 469.9 & 2.8 & 3565.9 & 3.4 & 5035.8 & 3.2 \\
\hline Tlaxcala & 505.1 & 1.0 & 628.0 & 0.6 & I 133.1 & 0.7 \\
\hline Veracruz & 3312.6 & 6.3 & 5916.6 & 5.7 & 9229.2 & 5.9 \\
\hline Yucatán & 983.7 & 1.9 & 1929.6 & 1.9 & 2913.4 & 1.9 \\
\hline Zacatecas & 792.7 & 1.5 & 847.7 & 0.8 & I 640.5 & I.I \\
\hline
\end{tabular}

Notas

I. El presupuesto ejercido en población no asegurada incluye los recursos de Ramo 12 (PAC, Progresa, IMSS-Solidaridad, Unidades Centrales, Organismos Descentralizados y Organos Desconcentrados), Ramo 33 y Aportaciones Estatales

2. El presupuesto ejercido en población asegurada incluye: IMSS, ISSSTE y Pemex

PAC: Programa de Ampliación de Cobertura

Progresa: Programa de Educación, Salud y Alimentación

IMSS: Instituto Mexicano del Seguro Social

ISSSTE: Instituto de Seguridad y Servicios Sociales de los Trabajadores del Estado

Pemex: Petróleos Mexicanos

Fuente: Secretaría de Salud. Dirección General de Información y Evaluación del Desempeño. Boletín de Información Estadística No. 2I, 200I,Volumen IV 


\section{Cuadro II \\ Distribución del PRESUPUESTO PÚBLICO EJERCIDO POR FONDO de financiamiento y entidad federativa. México, 2001 (MILLONES DE PESOS CORRIENTES)}

\begin{tabular}{|c|c|c|c|c|c|c|c|c|c|c|c|}
\hline \multirow{3}{*}{ Entidad federativa } & \multicolumn{7}{|c|}{ Población no asegurada } & \multicolumn{3}{|c|}{ Población asegurada } & \multirow{3}{*}{$\begin{array}{c}\text { Presupuesto } \\
\text { público total } \\
\text { ejercido }\end{array}$} \\
\hline & Transferencias & & Programa & & Transferencias directas & & & & & & \\
\hline & $\begin{array}{l}\text { indirectas de } \\
\text { ramo } 12^{*}\end{array}$ & $\begin{array}{c}\text { IMSS } \\
\text { Solidaridad }\end{array}$ & $\begin{array}{l}\text { de ampliación } \\
\text { de cobertura }\end{array}$ & Progresa & $\begin{array}{l}\text { a través de FASSA } \\
\quad \text { (ramo 33) }\end{array}$ & $\begin{array}{l}\text { Aportación } \\
\text { federal }^{\ddagger}\end{array}$ & $\begin{array}{c}\text { Aportación } \\
\text { estatal }\end{array}$ & IMSS & ISSSTE & Pemex & \\
\hline Nacional & 12269.2 & 3986.0 & I 168.7 & I 580.3 & 25770.5 & 44774.7 & 7507.1 & 84282.2 & 14353.6 & 5167.5 & I56 085.I \\
\hline Aguascalientes & 80.2 & 0.0 & 0.0 & 2.0 & 400.3 & 482.5 & 140.8 & 1017.8 & 106.1 & 6.1 & I 753.4 \\
\hline Baja California & 197.7 & 38.7 & 0.0 & 4.1 & 565.0 & 805.6 & $n d^{\S}$ & 2918.0 & 256.5 & 18.3 & 3998.4 \\
\hline Baja California Sur & 36.1 & 0.0 & 0.0 & 2.9 & 287.3 & 326.3 & 33.6 & 711.5 & 169.3 & 3.1 & 1243.9 \\
\hline Campeche & 58.2 & 59.6 & 14.8 & 24.1 & 391.6 & 548.3 & 67.0 & 539.1 & 98.3 & 202.5 & | $455 . \mid$ \\
\hline Coahuila & 181.8 & 120.4 & 0.0 & 11.0 & 504.4 & 817.6 & 119.5 & 3357.2 & 287.5 & 17.9 & 4599.7 \\
\hline Colima & 44.3 & 0.0 & 0.0 & 5.3 & 287.2 & 336.7 & 12.0 & 570.5 & 101.0 & 7.9 & 1028.2 \\
\hline Chiapas & 368.0 & 548.3 & 111.8 & 167.2 & I 179.0 & 2374.5 & 24.8 & 1004.6 & 262.1 & 39.8 & 3705.8 \\
\hline Chihuahua & 240.9 & 196.7 & 61.7 & 14.5 & 692.5 & I 206.4 & 6.0 & 3334.0 & 293.5 & 51.2 & 4891.1 \\
\hline Distrito Federal & 2963.8 & 121.7 & 43.3 & 0.0 & I 463.8 & 4592.7 & 2761.4 & 19357.5 & 8043.6 & 1216.0 & 35971.2 \\
\hline Durango & 121.1 & 163.8 & 26.2 & 15.5 & 538.7 & 865.3 & 16.1 & 1218.5 & 236.1 & 20.2 & 2356.3 \\
\hline Guanajuato & 448.7 & 0.0 & 21.1 & 66.7 & 966.1 & 1502.6 & 328.8 & 2834.7 & 217.2 & 270.5 & 5153.7 \\
\hline Guerrero & 365.8 & 0.0 & 80.4 & 142.9 & I 282.5 & 1871.6 & 20.4 & 1121.3 & 351.5 & 7.0 & 3371.7 \\
\hline Hidalgo & 295.3 & 263.2 & 105.8 & 78.7 & 698.2 & | $44 \mid .2$ & 36.8 & 1013.5 & 170.6 & 104.9 & 2767.1 \\
\hline Jalisco & 515.0 & 0.0 & 21.9 & 43.4 & 1692.8 & 2273.2 & I 037.5 & 6384.1 & 115.9 & 45.1 & 9855.8 \\
\hline México & 2349.6 & 0.0 & 62.8 & 108.2 & 3014.0 & 5534.6 & $15 \mid .5$ & 6813.9 & 272.1 & 27.4 & 12799.6 \\
\hline Michoacán & 419.5 & 295.3 & 41.9 & 88.2 & 907.3 & I $752 . \mid$ & 51.5 & 1686.8 & $4 \mid 4.1$ & 13.0 & 3917.4 \\
\hline Morelos & 203.8 & 0.0 & 0.0 & 15.2 & 479.7 & 698.8 & 105.2 & 1160.2 & 217.5 & 7.9 & 2189.6 \\
\hline Nayarit & 77.2 & 66.0 & 15.3 & 18.3 & 371.5 & 548.3 & 40.1 & 791.4 & 147.2 & 2.8 & I 529.8 \\
\hline Nuevo León & 296.6 & 0.0 & 0.0 & 12.1 & 773.0 & I 081.7 & 66.7 & 5648.7 & 111.9 & 165.5 & 7074.5 \\
\hline Oaxaca & 352.9 & 492.2 & 130.3 & 124.1 & I 043.5 & 2143.0 & 26.8 & 840.7 & 162.8 & 137.6 & 3310.9 \\
\hline Puebla & 481.8 & 327.9 & 130.6 & 104.9 & 821.5 & I 866.8 & 139.6 & 2753.7 & 96.6 & 72.3 & 4929.1 \\
\hline Querétaro & 132.8 & 0.0 & 26.9 & 32.7 & 483.1 & 675.4 & 36.9 & I 122.4 & 109.9 & 9.9 & 1954.6 \\
\hline Quintana Roo & 72.2 & 0.0 & 0.0 & 16.7 & 428.1 & 517.0 & 68.4 & 912.8 & 117.4 & 0.0 & 1615.6 \\
\hline San Luis Potosí & 195.0 & 242.9 & 47.6 & 72.4 & 525.9 & I 083.8 & 57.1 & I 48I.I & 208.1 & 57.2 & 2887.2 \\
\hline Sinaloa & 203.1 & 134.6 & 30.8 & 37.0 & 633.8 & I 039.4 & 227.8 & 2381.9 & 211.4 & 34.4 & 3894.8 \\
\hline Sonora & 176.0 & 0.0 & 0.0 & 19.9 & 766.4 & 962.3 & 337.3 & 2604.5 & 287.9 & 39.1 & 4231.0 \\
\hline Tabasco & 163.8 & 0.0 & 0.0 & 64.4 & 740.4 & 968.5 & 987.9 & $88 I .1$ & 136.4 & 673.6 & 3647.6 \\
\hline Tamaulipas & 222.1 & 99.9 & 0.0 & 22.0 & 929.3 & I 273.4 & 196.6 & 2393.9 & 448.3 & 723.7 & 5035.8 \\
\hline Tlaxcala & 104.6 & 0.0 & 0.0 & 12.4 & 356.1 & 473.1 & 32.0 & 550.1 & 76.2 & 1.8 & I I33.I \\
\hline Veracruz & 633.1 & 445.7 & I34.I & 165.0 & I 637.2 & 3015.1 & 297.5 & 4295.9 & 454.0 & I I 66.7 & 9229.2 \\
\hline Yucatán & 148.9 & 165.4 & 33.0 & 40.6 & 562.4 & 950.3 & 33.4 & I 880.8 & 28.5 & 20.3 & 2913.4 \\
\hline Zacatecas & 119.1 & 203.3 & 28.4 & 47.8 & 348.1 & 746.7 & 46.0 & 699.9 & 144.1 & 3.7 & 1640.5 \\
\hline
\end{tabular}

* Ramo 12 incluye Unidades Centrales, Organos Desconcentrados y Organismos Descentralizados. La distribución del Ramo 12 por entidad federativa se realizó de la siguiente manera: lo correspondiente a Unidades Centrales se distribuyó en función de la participación que cada entidad tiene en la población total nacional. El Hospital Manuel Gea González y el Hospital General de México se distribuye en función del lugar de procedencia de las consultas de primera vez. Los Institutos Nacionales, en función de los egresos hospitalarios. La Administración del Patrimonio de la Beneficencia Pública y el Hospital Juárez de México, en función de la población no asegurada.Y finalmente, el resto de Organismos Descentralizados y Organos Desconcentrados, con base en la participación de cada entidad en el total de población

₹ Las aportaciones federales incluyen: Ramo 12, IMSS-Solidaridad, PAC, Progresa y FASSA (Ramo 33)

$\S$ nd: no disponible

PAC: Programa de Ampliación de Cobertura

Progresa: Programa de Educación, Salud y Alimentación

FASSA: Fondo de Aportaciones para los Servicios de Salud

IMSS: Instituto Mexicano del Seguro Social

ISSSTE: Instituto de Seguridad y Servicios Sociales de los Trabajadores del Estado

Pemex: Petróleos Mexicanos

Fuente: Secretaría de Salud. Dirección General de Información y Evaluación del Desempeño. Boletín de Información Estadística No. 2I, 200I,Volumen IV 


\section{Cuadro III}

\section{DistribuCión Del PRESUPUESTO PÚBLICO EJERCIDO POR FONDO de financiamiento y entidad federativa. México, $200 \mathrm{I}$. (ESTRUCTURA PORCENTUAL)}

\begin{tabular}{|c|c|c|c|c|c|c|c|c|c|c|c|}
\hline \multirow[b]{2}{*}{ Entidad federativa } & \multicolumn{7}{|c|}{ Población no asegurada } & \multicolumn{3}{|c|}{ Población asegurada } & \multirow[b]{2}{*}{$\begin{array}{c}\text { Presupuesto } \\
\text { ejercido } \\
\text { total }\end{array}$} \\
\hline & $\begin{array}{l}\text { Transferencias } \\
\text { indirectas de } \\
\text { ramo I2* }\end{array}$ & $\begin{array}{c}\text { IMSS } \\
\text { Solidaridad }\end{array}$ & $\begin{array}{c}\text { Programa } \\
\text { de ampliación } \\
\text { de cobertura }\end{array}$ & Progresa & $\begin{array}{c}\text { Transferencias directas } \\
\text { a través de FASSA } \\
\text { (ramo 33) }\end{array}$ & $\begin{array}{l}\text { Aportación } \\
\text { federał }\end{array}$ & $\begin{array}{l}\text { Aportación } \\
\text { estatal }\end{array}$ & IMSS & ISSSTE & Pemex & \\
\hline Nacional & 100.0 & 100.0 & 100.0 & 100.0 & 100.0 & 100.0 & 100.0 & 100.0 & 100.0 & 100.0 & 100.0 \\
\hline Aguascalientes & 0.7 & 0.0 & 0.0 & 0.1 & 1.6 & 1.1 & 1.9 & 1.2 & 0.7 & 0.1 & 1.1 \\
\hline Baja California & 1.6 & 1.0 & 0.0 & 0.3 & 2.2 & 1.8 & $n d^{\S}$ & 3.5 & 1.8 & 0.4 & 2.6 \\
\hline Baja California Sur & 0.3 & 0.0 & 0.0 & 0.2 & 1.1 & 0.7 & 0.4 & 0.8 & 1.2 & 0.1 & 0.8 \\
\hline Campeche & 0.5 & 1.5 & 1.3 & 1.5 & 1.5 & 1.2 & 0.9 & 0.6 & 0.7 & 3.9 & 0.9 \\
\hline Coahuila & 1.5 & 3.0 & 0.0 & 0.7 & 2.0 & 1.8 & 1.6 & 4.0 & 2.0 & 0.3 & 2.9 \\
\hline Colima & 0.4 & 0.0 & 0.0 & 0.3 & 1.1 & 0.8 & 0.2 & 0.7 & 0.7 & 0.2 & 0.7 \\
\hline Chiapas & 3.0 & 13.8 & 9.6 & 10.6 & 4.6 & 5.3 & 0.3 & 1.2 & 1.8 & 0.8 & 2.4 \\
\hline Chihuahua & 2.0 & 4.9 & 5.3 & 0.9 & 2.7 & 2.7 & 0.1 & 4.0 & 2.0 & 1.0 & 3.1 \\
\hline Distrito Federal & 24.2 & 3.1 & 3.7 & 0.0 & 5.7 & 10.3 & 36.8 & 23.0 & 56.0 & 23.5 & 23.0 \\
\hline Durango & 1.0 & 4.1 & 2.2 & 1.0 & 2.1 & 1.9 & 0.2 & 1.4 & 1.6 & 0.4 & 1.5 \\
\hline Guanajuato & 3.7 & 0.0 & 1.8 & 4.2 & 3.7 & 3.4 & 4.4 & 3.4 & 1.5 & 5.2 & 3.3 \\
\hline Guerrero & 3.0 & 0.0 & 6.9 & 9.0 & 5.0 & 4.2 & 0.3 & 1.3 & 2.4 & 0.1 & 2.2 \\
\hline Hidalgo & 2.4 & 6.6 & 9.1 & 5.0 & 2.7 & 3.2 & 0.5 & 1.2 & 1.2 & 2.0 & 1.8 \\
\hline Jalisco & 4.2 & 0.0 & 1.9 & 2.7 & 6.6 & 5.1 & 13.8 & 7.6 & 0.8 & 0.9 & 6.3 \\
\hline México & 19.2 & 0.0 & 5.4 & 6.8 & 11.7 & 12.4 & 2.0 & 8.1 & 1.9 & 0.5 & 8.2 \\
\hline Michoacán & 3.4 & 7.4 & 3.6 & 5.6 & 3.5 & 3.9 & 0.7 & 2.0 & 2.9 & 0.3 & 2.5 \\
\hline Morelos & 1.7 & 0.0 & 0.0 & 1.0 & 1.9 & 1.6 & 1.4 & 1.4 & 1.5 & 0.2 & 1.4 \\
\hline Nayarit & 0.6 & 1.7 & 1.3 & 1.2 & 1.4 & 1.2 & 0.5 & 0.9 & 1.0 & 0.1 & 1.0 \\
\hline Nuevo León & 2.4 & 0.0 & 0.0 & 0.8 & 3.0 & 2.4 & 0.9 & 6.7 & 0.8 & 3.2 & 4.5 \\
\hline Oaxaca & 2.9 & 12.3 & 11.2 & 7.8 & 4.0 & 4.8 & 0.4 & 1.0 & I.I & 2.7 & 2.1 \\
\hline Puebla & 3.9 & 8.2 & 11.2 & 6.6 & 3.2 & 4.2 & 1.9 & 3.3 & 0.7 & 1.4 & 3.2 \\
\hline Querétaro & 1.1 & 0.0 & 2.3 & 2.1 & 1.9 & 1.5 & 0.5 & 1.3 & 0.8 & 0.2 & 1.3 \\
\hline Quintana Roo & 0.6 & 0.0 & 0.0 & 1.1 & 1.7 & 1.2 & 0.9 & 1.1 & 0.8 & 0.0 & 1.0 \\
\hline San Luis Potosí & 1.6 & 6.1 & 4.1 & 4.6 & 2.0 & 2.4 & 0.8 & 1.8 & 1.4 & 1.1 & 1.8 \\
\hline Sinaloa & 1.7 & 3.4 & 2.6 & 2.3 & 2.5 & 2.3 & 3.0 & 2.8 & 1.5 & 0.7 & 2.5 \\
\hline Sonora & 1.4 & 0.0 & 0.0 & 1.3 & 3.0 & 2.1 & 4.5 & 3.1 & 2.0 & 0.8 & 2.7 \\
\hline Tabasco & 1.3 & 0.0 & 0.0 & 4.1 & 2.9 & 2.2 & 13.2 & 1.0 & 1.0 & 13.0 & 2.3 \\
\hline Tamaulipas & 1.8 & 2.5 & 0.0 & 1.4 & 3.6 & 2.8 & 2.6 & 2.8 & 3.1 & 14.0 & 3.2 \\
\hline Tlaxcala & 0.9 & 0.0 & 0.0 & 0.8 & 1.4 & 1.1 & 0.4 & 0.7 & 0.5 & 0.0 & 0.7 \\
\hline Veracruz & 5.2 & 11.2 & 11.5 & 10.4 & 6.4 & 6.7 & 4.0 & 5.1 & 3.2 & 22.6 & 5.9 \\
\hline Yucatán & 1.2 & 4.2 & 2.8 & 2.6 & 2.2 & 2.1 & 0.4 & 2.2 & 0.2 & 0.4 & 1.9 \\
\hline Zacatecas & 1.0 & 5.1 & 2.4 & 3.0 & 1.4 & 1.7 & 0.6 & 0.8 & 1.0 & 0.1 & 1.1 \\
\hline
\end{tabular}

* Ramo 12 incluye Unidades Centrales, Organos Desconcentrados y Organismos Descentralizados. La distribución del Ramo 12 por entidad federativa se realizó de la siguiente manera: lo correspondiente a Unidades Centrales se distribuyó en función de la participación que cada entidad tiene en la población total nacional. El Hospital Manuel Gea González y el Hospital General de México se distribuye en función del lugar de procedencia de las consultas de primera vez. Los Institutos Nacionales en función de los egresos hospitalarios. La Administración del Patrimonio de la Beneficencia Pública y el Hospital Juárez de México, en función de la población no asegurada.Y finalmente, el resto de Organismos Descentralizados y Organos Desconcentrados, con base en la participación de cada entidad en el total de población

₹ Las aportaciones federales incluyen: Ramo 12, IMSS-Solidaridad, PAC, Progresa y FASSA (Ramo 33)

$\S$ nd: no disponible

PAC: Programa de Ampliación de Cobertura

Progresa: Programa de Educación, Salud y Alimentación

FASSA: Fondo de Aportaciones para los Servicios de Salud

IMSS: Instituto Mexicano del Seguro Social

ISSSTE: Instituto de Seguridad y Servicios Sociales de los Trabajadores del Estado

Pemex: Petróleos Mexicanos

Fuente: Secretaría de Salud. Dirección General de Información y Evaluación del Desempeño. Boletín de Información Estadística No. 2I, 200I,Volumen IV 


\section{Cuadro IV \\ Distribución del PResupuesto público ejercido PARA población No ASEguRAda POR FONDO de finanCIAMIENTO Y ENTIDAD federativa. MéXICO, 200 I (ESTRUCTURA PORCENTUAL)}

\begin{tabular}{|c|c|c|c|c|c|c|c|}
\hline Entidad federativa & $\begin{array}{c}\text { Transferencias indirectas } \\
\text { de ramo } 12^{*}\end{array}$ & $\begin{array}{c}\text { IMSS } \\
\text { Solidaridad }\end{array}$ & $\begin{array}{c}\text { Programa de ampliación } \\
\text { de cobertura }\end{array}$ & Progresa & $\begin{array}{l}\text { Transferencias directas a través } \\
\text { de FASSA (ramo 33) }\end{array}$ & $\begin{array}{l}\text { Aportación } \\
\text { Estatal }\end{array}$ & $\begin{array}{l}\text { Total (millones de } \\
\text { pesos corrientes) }\end{array}$ \\
\hline Nacional & 23.5 & 7.6 & 2.2 & 3.0 & 49.3 & 14.4 & 52281.8 \\
\hline Aguascalientes & 12.9 & 0.0 & 0.0 & 0.3 & 64.2 & 22.6 & 623.3 \\
\hline Baja California & 24.5 & 4.8 & 0.0 & 0.5 & 70.1 & 0.0 & 805.6 \\
\hline Baja California Sur & 10.0 & 0.0 & 0.0 & 0.8 & 79.8 & 9.3 & 359.9 \\
\hline Campeche & 9.5 & 9.7 & 2.4 & 3.9 & 63.7 & 10.9 & 615.3 \\
\hline Coahuila & 19.4 & 12.9 & 0.0 & 1.2 & 53.8 & 12.8 & 937.1 \\
\hline Colima & 12.7 & 0.0 & 0.0 & 1.5 & 82.3 & 3.5 & 348.8 \\
\hline Chiapas & 15.3 & 22.9 & 4.7 & 7.0 & 49.1 & 1.0 & 2399.3 \\
\hline Chihuahua & 19.9 & 16.2 & 5.1 & 1.2 & 57.1 & 0.5 & I 212.4 \\
\hline Distrito Federal & 40.3 & 1.7 & 0.6 & 0.0 & 19.9 & 37.5 & 7354.1 \\
\hline Durango & 13.7 & 18.6 & 3.0 & 1.8 & 61.1 & 1.8 & 881.5 \\
\hline Guanajuato & 24.5 & 0.0 & 1.2 & 3.6 & 52.8 & 18.0 & 1831.3 \\
\hline Guerrero & 19.3 & 0.0 & 4.2 & 7.6 & 67.8 & I.I & 1891.9 \\
\hline Hidalgo & 20.0 & 17.8 & 7.2 & 5.3 & 47.2 & 2.5 & I 478.0 \\
\hline Jalisco & 15.6 & 0.0 & 0.7 & 1.3 & 51.1 & 31.3 & 3310.7 \\
\hline México & 41.3 & 0.0 & 1.1 & 1.9 & 53.0 & 2.7 & 5686.1 \\
\hline Michoacán & 23.3 & 16.4 & 2.3 & 4.9 & 50.3 & 2.9 & 1803.6 \\
\hline Morelos & 25.3 & 0.0 & 0.0 & 1.9 & 59.7 & 13.1 & 804.0 \\
\hline Nayarit & 13.1 & 11.2 & 2.6 & 3.1 & 63.1 & 6.8 & 588.4 \\
\hline Nuevo León & 25.8 & 0.0 & 0.0 & 1.1 & 67.3 & 5.8 & I I 48.4 \\
\hline Oaxaca & 16.3 & 22.7 & 6.0 & 5.7 & 48.1 & 1.2 & 2169.8 \\
\hline Puebla & 24.0 & 16.3 & 6.5 & 5.2 & 40.9 & 7.0 & 2006.4 \\
\hline Querétaro & 18.6 & 0.0 & 3.8 & 4.6 & 67.8 & 5.2 & 712.4 \\
\hline Quintana Roo & 12.3 & 0.0 & 0.0 & 2.8 & 73.1 & 11.7 & 585.4 \\
\hline San Luis Potosí & 17.1 & 21.3 & 4.2 & 6.3 & 46.1 & 5.0 & 1140.9 \\
\hline Sinaloa & 16.0 & 10.6 & 2.4 & 2.9 & 50.0 & 18.0 & I 267.I \\
\hline Sonora & 13.5 & 0.0 & 0.0 & 1.5 & 59.0 & 26.0 & I 299.6 \\
\hline Tabasco & 8.4 & 0.0 & 0.0 & 3.3 & 37.8 & 50.5 & I 956.4 \\
\hline Tamaulipas & 15.1 & 6.8 & 0.0 & 1.5 & 63.2 & 13.4 & 1469.9 \\
\hline Tlaxcala & 20.7 & 0.0 & 0.0 & 2.5 & 70.5 & 6.3 & 505.1 \\
\hline Veracruz & 19.1 & 13.5 & 4.0 & 5.0 & 49.4 & 9.0 & 3312.6 \\
\hline Yucatán & 15.1 & 16.8 & 3.4 & 4.1 & 57.2 & 3.4 & 983.7 \\
\hline Zacatecas & 15.0 & 25.6 & 3.6 & 6.0 & 43.9 & 5.8 & 792.7 \\
\hline
\end{tabular}

\footnotetext{
* Ramo 12 incluye Unidades Centrales, Organos Desconcentrados y Organismos Descentralizados. La distribución del Ramo 12 por entidad federativa se realizó de la siguiente manera: lo correspondiente a Unidades Centrales se distribuyó en función de la participación que cada entidad tiene en la población total nacional. El Hospital Manuel Gea González y el Hospital General de México se distribuye en función del lugar de procedencia de las consultas de primera vez. Los Institutos Nacionales en función de los egresos hospitalarios. La Administración del Patrimonio de la Beneficencia Pública y el Hospital Juárez de México en función de la población no asegurada.Y, finalmente, el resto de Organismos Descentralizados y Organos Desconcentrados con base en la participación de cada entidad en el total de población
}

Progresa: Programa de Educación, Salud y Alimentación

FASSA: Fondo de Aportaciones para los Servicios de Salud

Fuente: Secretaría de Salud. Dirección General de Información y Evaluación del Desempeño. Boletín de Información Estadística No. 2I, 200I,Volumen IV 


\section{Cuadro V \\ Distribución del presupuesto público ejercido, por RUbro de gasto y entidad federativa. México, 200 I. (MILLONES DE PESOS CORRIENTES)}

\begin{tabular}{|c|c|c|c|c|c|c|c|c|c|c|c|c|}
\hline \multirow[b]{2}{*}{ Entidad federativa } & \multicolumn{4}{|c|}{ Total } & \multicolumn{4}{|c|}{ Población no asegurada } & \multicolumn{4}{|c|}{ Población asegurada } \\
\hline & Personal & Operación & Infraestructura & Resto & Personal & Operación & Infraestructura & Resto & Personal & Operación & Infraestructura & Total \\
\hline Nacional & 105678.6 & 47045.3 & 3176.3 & 184.8 & 31786.5 & 18719.7 & 1761.1 & 14.5 & 73892.2 & 28325.6 & | 415.2 & 170.4 \\
\hline Aguascalientes & | 209.4 & 504.1 & 37.5 & 2.4 & 399.8 & 190.2 & 33.2 & 0.1 & 809.6 & 313.9 & 4.3 & 2.3 \\
\hline Baja California & 2922.6 & I 042.5 & 29.4 & 3.9 & 559.1 & 243.5 & 2.6 & 0.4 & 2363.5 & 799.1 & 26.8 & 3.5 \\
\hline Baja California Sur & 938.0 & 275.5 & 26.3 & 4.1 & 262.1 & 77.9 & 19.9 & 0.1 & 676.0 & 197.5 & 6.5 & 4.0 \\
\hline Campeche & 1053.8 & 361.6 & 35.4 & 4.3 & 435.9 & 165.9 & 13.4 & 0.1 & 617.9 & 195.8 & 22.0 & 4.2 \\
\hline Coahuila & 3246.4 & | 239.8 & 108.3 & 5.2 & 600.9 & 260.4 & 75.5 & 0.3 & 2645.6 & 979.5 & 32.8 & 4.8 \\
\hline Colima & 799.5 & 216.8 & 10.1 & 1.8 & 280.0 & 65.6 & 3.1 & 0.1 & 519.5 & 151.2 & 7.0 & 1.7 \\
\hline Chiapas & 2526.6 & I II4.I & 50.5 & 14.6 & I 544.9 & 825.5 & 28.3 & 0.6 & 981.7 & 288.6 & 22.2 & 14.1 \\
\hline Chihuahua & 3534.0 & 1252.9 & 95.3 & 8.8 & 833.9 & 349.4 & 28.6 & 0.5 & 2700.2 & 903.5 & 66.7 & 8.4 \\
\hline Distrito Federal & 22852.7 & 12763.8 & 336.8 & 17.8 & 3515.4 & 3739.2 & 98.3 & 1.2 & 19337.3 & 9024.6 & 238.6 & 16.6 \\
\hline Durango & I 730.7 & 596.7 & 23.4 & 5.5 & 643.5 & 225.4 & 12.4 & 0.2 & I 087.2 & 371.3 & 11.0 & 5.3 \\
\hline Guanajuato & 3350.8 & 1636.2 & 159.6 & 7.0 & 1016.2 & 669.4 & 145.0 & 0.7 & 2334.5 & 966.8 & 14.6 & 6.3 \\
\hline Guerrero & 2346.9 & 975.8 & 39.6 & 9.5 & 1219.1 & 651.8 & 20.5 & 0.5 & I 127.8 & 324.0 & 19.0 & 9.0 \\
\hline Hidalgo & 1930.2 & 803.5 & 30.9 & 2.5 & 967.4 & 491.8 & 18.6 & 0.3 & 962.9 & 311.7 & 12.3 & 2.2 \\
\hline Jalisco & 7145.9 & 2512.8 & 192.7 & 4.4 & 2400.8 & 754.0 & 155.0 & 0.9 & 4745.1 & I 758.8 & 37.7 & 3.5 \\
\hline México & 8004.4 & 4279.5 & 513.4 & 2.3 & 2838.9 & 2661.7 & 183.6 & 1.9 & 5165.5 & 1617.8 & 329.8 & 0.4 \\
\hline Michoacán & 2624.5 & | 208.9 & 70.7 & 13.3 & I 036.5 & 710.3 & 56.1 & 0.6 & | 588.0 & 498.6 & 14.6 & 12.7 \\
\hline Morelos & I 540.3 & 631.7 & 14.9 & 2.7 & 496.8 & 302.6 & 4.4 & 0.2 & I 043.5 & 329.1 & 10.6 & 2.5 \\
\hline Nayarit & I 128.4 & 356.1 & 41.4 & 4.0 & 410.5 & 157.6 & 20.1 & 0.1 & 717.9 & 198.4 & 21.3 & 3.8 \\
\hline Nuevo León & 4943.4 & 2099.4 & 29.5 & 2.2 & 779.4 & 356.7 & 11.7 & 0.6 & 4163.9 & I 742.7 & 17.8 & 1.7 \\
\hline Oaxaca & 2214.0 & 975.0 & 113.1 & 8.7 & I 354.4 & 721.2 & 93.7 & 0.5 & 859.7 & 253.8 & 19.4 & 8.2 \\
\hline Puebla & 3213.4 & I 569.6 & 143.5 & 2.6 & I 127.5 & 799.8 & 78.3 & 0.7 & 2085.9 & 769.8 & 65.1 & 1.9 \\
\hline Querétaro & I $4 \mid 2.7$ & 496.0 & 44.5 & 1.4 & 515.8 & 175.5 & 20.8 & 0.2 & 896.9 & 320.5 & 23.6 & 1.2 \\
\hline Quintana Roo & I 205.3 & 361.1 & 43.5 & 5.7 & 428.9 & 147.6 & 8.9 & 0.1 & 776.5 & 213.5 & 34.6 & 5.6 \\
\hline San Luis Potosí & 1838.0 & 884.3 & 158.2 & 6.6 & 706.8 & 419.4 & 14.4 & 0.3 & 1131.3 & 465.0 & 143.8 & 6.3 \\
\hline Sinaloa & 2668.0 & 1151.4 & 71.0 & 4.5 & 819.8 & 394.4 & 52.6 & 0.4 & | 848.| & 757.0 & 18.4 & 4.1 \\
\hline Sonora & 2884.0 & I 308.0 & 32.5 & 6.5 & 874.4 & 405.7 & 19.1 & 0.3 & 2009.6 & 902.3 & 13.4 & 6.2 \\
\hline Tabasco & 2627.9 & 900.8 & 114.4 & 4.5 & 1416.9 & 458.7 & 80.6 & 0.3 & 1211.0 & 442.1 & 33.9 & 4.3 \\
\hline Tamaulipas & 3645.3 & 1318.5 & 63.9 & 8.1 & 1143.5 & 311.3 & 14.7 & 0.4 & 2501.8 & I 007.2 & 49.2 & 7.7 \\
\hline Tlaxcala & 853.0 & 269.4 & 10.2 & 0.5 & 336.3 & 163.2 & 5.4 & 0.1 & 516.7 & 106.2 & 4.8 & 0.4 \\
\hline Veracruz & 6076.9 & 2644.4 & 495.9 & 12.1 & 1615.5 & 1280.8 & 415.4 & 1.0 & $446 I .4$ & 1363.6 & 80.6 & 11.1 \\
\hline Yucatán & 2063.1 & 823.6 & 23.2 & 3.5 & 682.2 & 287.9 & 13.4 & 0.2 & 1380.8 & 535.7 & 9.8 & 3.2 \\
\hline Zacatecas & I I 48.5 & 471.6 & 16.8 & 3.6 & 523.4 & 255.4 & 13.7 & 0.2 & 625.1 & 216.2 & 3.1 & 3.4 \\
\hline
\end{tabular}

Notas

I. El presupuesto para población no asegurada incluye los recursos de ramo 12 (Unidades Centrales, Organos Desconcentrados y Organismos Descentralizados. La distribución del Ramo 12 por entidad federativa se realizó de la siguiente manera: lo correspondiente a Unidades Centrales se distribuyó en función de la participación que cada entidad tiene en la población total nacional. El Hospital Manuel Gea González y el Hospital General de México se distribuye en función del lugar de procedencia de las consultas de primera vez. Los Institutos Nacionales, en función de los egresos hospitalarios. La Administración del Patrimonio de la Beneficencia Pública y el Hospital Juárez de México, en función de la población no asegurada.Y finalmente, el resto de Organismos Descentralizados y Organos Desconcentrados, con base en la participación de cada entidad en el total de población) FASSA (Ramo 33) y aportaciones estatales

2. Presupuesto en población asegurada incluye: IMSS, ISSSTE y Pemex

3. El gasto de personal incluye solamente capítulo 1000 . El gasto de operación incluye capítulos 2000,3000 y 4000 . El gasto de infraestructura incluye los capítulos 5000 y 6000 . Finalmente, el resto incluye el capítulo 7000

FASSA: Fondo de Aportaciones para los Servicios de Salud

IMSS: Instituto Mexicano del Seguro Social

ISSSTE: Instituto de Seguridad y Servicios Sociales de los Trabajadores del Estado

Pemex: Petróleos Mexicanos

Fuente: Secretaría de Salud. Dirección General de Información y Evaluación del Desempeño. Boletín de Información Estadística No. 2I, 200I,Volumen IV 


\section{Cuadro VI \\ Distribución del presupuesto público ejercido, por RUbro de gasto y entidad federativa. México, 200 I. (ESTRUCTURA PORCENTUAL)}

\begin{tabular}{|c|c|c|c|c|c|c|c|c|c|c|c|c|}
\hline \multirow[b]{2}{*}{ Entidad federativa } & \multicolumn{4}{|c|}{ Total } & \multicolumn{4}{|c|}{ Población no asegurada } & \multicolumn{4}{|c|}{ Población asegurada } \\
\hline & Personal & Operación & Infraestructura & Resto & Personal & Operación & Infraestructura & Resto & Personal & Operación & Infraestructura & Total \\
\hline Nacional & 67.7 & 30.1 & 2.0 & 0.1 & 60.80 & 35.81 & 3.37 & 0.03 & 71.2 & 27.3 & 1.4 & 0.2 \\
\hline Aguascalientes & 69.0 & 28.8 & 2.1 & 0.1 & 64.14 & 30.51 & 5.32 & 0.02 & 71.6 & 27.8 & 0.4 & 0.2 \\
\hline Baja California & 73.1 & 26.1 & 0.7 & 0.1 & 69.41 & 30.22 & 0.32 & 0.05 & 74.0 & 25.0 & 0.8 & 0.1 \\
\hline Baja California Sur & 75.4 & 22.1 & 2.1 & 0.3 & 72.81 & 21.65 & 5.52 & 0.02 & 76.5 & 22.3 & 0.7 & 0.5 \\
\hline Campeche & 72.4 & 24.9 & 2.4 & 0.3 & 70.85 & 26.96 & 2.17 & 0.02 & 73.6 & 23.3 & 2.6 & 0.5 \\
\hline Coahuila & 70.6 & 27.0 & 2.4 & 0.1 & 64.12 & 27.79 & 8.06 & 0.04 & 72.2 & 26.7 & 0.9 & 0.1 \\
\hline Colima & 77.8 & 21.1 & 1.0 & 0.2 & 80.28 & 18.82 & 0.88 & 0.02 & 76.5 & 22.3 & 1.0 & 0.3 \\
\hline Chiapas & 68.2 & 30.1 & 1.4 & 0.4 & 64.39 & 34.41 & 1.18 & 0.02 & 75.1 & 22.1 & 1.7 & 1.1 \\
\hline Chihuahua & 72.3 & 25.6 & 1.9 & 0.2 & 68.78 & 28.82 & 2.36 & 0.04 & 73.4 & 24.6 & 1.8 & 0.2 \\
\hline Distrito Federal & 63.5 & 35.5 & 0.9 & 0.0 & 47.80 & 50.84 & 1.34 & 0.02 & 67.6 & 31.5 & 0.8 & 0.1 \\
\hline Durango & 73.4 & 25.3 & 1.0 & 0.2 & 73.00 & 25.57 & 1.40 & 0.02 & 73.7 & 25.2 & 0.7 & 0.4 \\
\hline Guanajuato & 65.0 & 31.7 & 3.1 & 0.1 & 55.49 & 36.55 & 7.92 & 0.04 & 70.3 & 29.1 & 0.4 & 0.2 \\
\hline Guerrero & 69.6 & 28.9 & 1.2 & 0.3 & 64.44 & 34.45 & 1.09 & 0.02 & 76.2 & 21.9 & 1.3 & 0.6 \\
\hline Hidalgo & 69.8 & 29.0 & 1.1 & 0.1 & 65.45 & 33.27 & 1.26 & 0.02 & 74.7 & 24.2 & 1.0 & 0.2 \\
\hline Jalisco & 72.5 & 25.5 & 2.0 & 0.0 & 72.52 & 22.77 & 4.68 & 0.03 & 72.5 & 26.9 & 0.6 & 0.1 \\
\hline México & 62.5 & 33.4 & 4.0 & 0.0 & 49.93 & 46.81 & 3.23 & 0.03 & 72.6 & 22.7 & 4.6 & 0.0 \\
\hline Michoacán & 67.0 & 30.9 & 1.8 & 0.3 & 57.47 & 39.39 & 3.11 & 0.03 & 75.1 & 23.6 & 0.7 & 0.6 \\
\hline Morelos & 70.3 & 28.8 & 0.7 & 0.1 & 61.80 & 37.63 & 0.54 & 0.03 & 75.3 & 23.8 & 0.8 & 0.2 \\
\hline Nayarit & 73.8 & 23.3 & 2.7 & 0.3 & 69.77 & 26.79 & 3.41 & 0.02 & 76.3 & 21.1 & 2.3 & 0.4 \\
\hline Nuevo León & 69.9 & 29.7 & 0.4 & 0.0 & 67.87 & 31.06 & 1.02 & 0.05 & 70.3 & 29.4 & 0.3 & 0.0 \\
\hline Oaxaca & 66.9 & 29.4 & 3.4 & 0.3 & 62.42 & 33.24 & 4.32 & 0.02 & 75.3 & 22.2 & 1.7 & 0.7 \\
\hline Puebla & 65.2 & 31.8 & 2.9 & 0.1 & 56.19 & 39.86 & 3.90 & 0.04 & 71.4 & 26.3 & 2.2 & 0.1 \\
\hline Querétaro & 72.3 & 25.4 & 2.3 & 0.1 & 72.41 & 24.63 & 2.93 & 0.03 & 72.2 & 25.8 & 1.9 & 0.1 \\
\hline Quintana Roo & 74.6 & 22.4 & 2.7 & 0.4 & 73.26 & 25.21 & 1.51 & 0.02 & 75.4 & 20.7 & 3.4 & 0.5 \\
\hline San Luis Potosí & 63.7 & 30.6 & 5.5 & 0.2 & 61.95 & 36.76 & 1.27 & 0.03 & 64.8 & 26.6 & 8.2 & 0.4 \\
\hline Sinaloa & 68.5 & 29.6 & 1.8 & 0.1 & 64.70 & 31.12 & 4.15 & 0.03 & 70.3 & 28.8 & 0.7 & 0.2 \\
\hline Sonora & 68.2 & 30.9 & 0.8 & 0.2 & 67.29 & 31.22 & 1.47 & 0.03 & 68.6 & 30.8 & 0.5 & 0.2 \\
\hline Tabasco & 72.0 & 24.7 & 3.1 & 0.1 & 72.42 & 23.44 & 4.12 & 0.01 & 71.6 & 26.1 & 2.0 & 0.3 \\
\hline Tamaulipas & 72.4 & 26.2 & 1.3 & 0.2 & 77.79 & 21.18 & 1.00 & 0.03 & 70.2 & 28.2 & 1.4 & 0.2 \\
\hline Tlaxcala & 75.3 & 23.8 & 0.9 & 0.0 & 66.58 & 32.32 & 1.07 & 0.03 & 82.3 & 16.9 & 0.8 & 0.1 \\
\hline Veracruz & 65.8 & 28.7 & 5.4 & 0.1 & 48.77 & 38.66 & 12.54 & 0.03 & 75.4 & 23.0 & 1.4 & 0.2 \\
\hline Yucatán & 70.8 & 28.3 & 0.8 & 0.1 & 69.35 & 29.26 & 1.36 & 0.02 & 71.6 & 27.8 & 0.5 & 0.2 \\
\hline Zacatecas & 70.0 & 28.7 & 1.0 & 0.2 & 66.03 & 32.22 & 1.73 & 0.03 & 73.7 & 25.5 & 0.4 & 0.4 \\
\hline
\end{tabular}

Notas

I. El presupuesto para población no asegurada incluye los recursos de ramo 12 (Unidades Centrales, Organos Desconcentrados y Organismos Descentralizados. La distribución del Ramo 12 por entidad federativa se realizó de la siguiente manera: lo correspondiente a Unidades Centrales se distribuyó en función de la participación que cada entidad tiene en la población total nacional. El Hospital Manuel Gea González y el Hospital General de México se distribuye en función del lugar de procedencia de las consultas de primera vez. Los Institutos Nacionales, en función de los egresos hospitalarios. La Administración del Patrimonio de la Beneficencia Pública y el Hospital Juárez de México, en función de la población no asegurada. Y finalmente, el resto de Organismos Descentralizados y Organos Desconcentrados, con base en la participación de cada entidad en el total de población) FASSA (Ramo 33) y aportaciones estatales

2. Presupuesto en población asegurada incluye: IMSS, ISSSTE y Pemex

3. El gasto de personal incluye solamente capítulo 1000 . El gasto de operación incluye capítulos 2000,3000 y 4000 . El gasto de infraestructura incluye los capítulos 5000 y 6000 . Finalmente, el resto incluye el capítulo 7000

FASSA: Fondo de Aportaciones para los Servicios de Salud

IMSS: Instituto Mexicano del Seguro Social

ISSSTE: Instituto de Seguridad y Servicios Sociales de los Trabajadores del Estado

Pemex: Petróleos Mexicanos

Fuente: Secretaría de Salud. Dirección General de Información y Evaluación del Desempeño. Boletín de Información Estadística No. 2I, 200I,Volumen IV 
Cuadro VII

Distribución de las APORTACIONES ESTATALES, POR RUBRo Y ENTIDAd federativa. MÉxico, 200 I (ESTRUCTURA PORCENTUAL)

Total

Entidad federativa Personal Operación Infraestructura (millones de pesos)

\begin{tabular}{|c|c|c|c|c|}
\hline Nacional & 61.3 & 24.6 & I4. I & 7507060.6 \\
\hline Aguascalientes & 33.9 & 52.9 & 13.2 & | 40807.3 \\
\hline Baja California & nd & nd & nd & nd \\
\hline Baja California Sur & 25.9 & 41.3 & 32.8 & 33590.0 \\
\hline Campeche & 44.5 & 51.5 & 4.0 & 66968.0 \\
\hline Coahuila & 32.3 & 8.3 & 59.4 & 119523.0 \\
\hline Colima & 66.2 & 17.0 & 16.7 & 12046.1 \\
\hline Chiapas & 83.5 & 10.2 & 6.2 & 24810.0 \\
\hline Chihuahua & 0.0 & 21.8 & 78.2 & 6019.0 \\
\hline Distrito Federal & 68.2 & 28.8 & 3.0 & $276 \mid 421.0$ \\
\hline Durango & 53.0 & 43.4 & 3.6 & 16149.6 \\
\hline Guanajuato & 31.9 & 30.1 & 38.0 & 328776.2 \\
\hline Guerrero & 88.9 & 5.2 & 5.9 & 20366.6 \\
\hline Hidalgo & 68.2 & 26.3 & 5.5 & 36831.9 \\
\hline Jalisco & 78.9 & 10.9 & 10.1 & 1037513.0 \\
\hline México & 0.0 & 22.0 & 78.0 & 151505.1 \\
\hline Michoacán & 47.8 & 23.9 & 28.3 & 51462.5 \\
\hline Morelos & 44.4 & 55.6 & 0.0 & 105214.1 \\
\hline Nayarit & 22.9 & 34.3 & 42.8 & 40065.2 \\
\hline Nuevo León & 71.1 & 27.9 & 1.0 & 66678.8 \\
\hline Oaxaca & 0.0 & 0.0 & 100.0 & 26837.2 \\
\hline Puebla & 43.6 & 26.0 & 30.4 & 139607.5 \\
\hline Querétaro & 100.0 & 0.0 & 0.0 & 36937.1 \\
\hline Quintana Roo & 56.6 & 38.2 & 5.2 & 68411.9 \\
\hline San Luis Potosí & 10.5 & 89.3 & 0.2 & 57070.3 \\
\hline Sinaloa & 54.0 & 29.2 & 16.7 & 227758.1 \\
\hline Sonora & 65.3 & 32.2 & 2.5 & 337285.8 \\
\hline Tabasco & 75.0 & 19.9 & 5.0 & 987903.0 \\
\hline Tamaulipas & 84.7 & 13.3 & 2.0 & 196568.0 \\
\hline Tlaxcala & 72.9 & 27.1 & 0.0 & 32000.0 \\
\hline Veracruz & 0.0 & 0.0 & 100.0 & 297497.9 \\
\hline Yucatán & 58.7 & 41.3 & 0.0 & 33420.0 \\
\hline Zacatecas & 48.7 & 34.4 & 16.9 & 46016.5 \\
\hline
\end{tabular}

Nota

I. El gasto de personal incluye solamente capítulo 1000. El gasto de operación incluye capítulos 2000,3000 y 4000 . El gasto de infraestructura incluye los capítulos 5000 y 6000

nd: no disponible

Fuente: Secretaría de Salud. Dirección General de Información y Evaluación del Desempeño. Boletín de Información Estadística No. 21, 200I, Volumen IV

\section{Cuadro VIII \\ Presupuesto ejercido en programas de combate a la pobreza. México, 200 I (ESTRUCTURA PORCENTUAL)}

$\begin{array}{cccc} & \text { Total (millones } & \text { Programa de } & \\ \text { Entidad federativa } & \text { de pesos } & \text { ampliación } & \text { IMSS } \\ \text { corrientes) } & \text { de cobertura } & \text { Progresa } & \text { Solidaridad }\end{array}$

$\begin{array}{lllll}\text { Nacional } & 6.735 .0 & 17.4 & 23.5 & 59.2\end{array}$

\begin{tabular}{|c|c|c|c|c|}
\hline Aguascalientes & 2.0 & 0.0 & 100.0 & 0.0 \\
\hline Baja California & 42.8 & 0.0 & 9.6 & 90.4 \\
\hline Baja California Sur & 2.9 & 0.0 & 100.0 & 0.0 \\
\hline Campeche & 98.5 & 15.0 & 24.5 & 60.5 \\
\hline Coahuila & 131.4 & 0.0 & 8.4 & 91.6 \\
\hline Colima & 5.3 & 0.0 & 100.0 & 0.0 \\
\hline Chiapas & 827.4 & 13.5 & 20.2 & 66.3 \\
\hline Chihuahua & 273.0 & 22.6 & 5.3 & 72.1 \\
\hline Distrito Federal & 165.1 & 26.3 & 0.0 & 73.7 \\
\hline Durango & 205.6 & 12.8 & 7.5 & 79.7 \\
\hline Guanajuato & 87.8 & 24.0 & 76.0 & 0.0 \\
\hline Guerrero & 223.3 & 36.0 & 64.0 & 0.0 \\
\hline Hidalgo & 447.7 & 23.6 & 17.6 & 58.8 \\
\hline Jalisco & 65.4 & 33.6 & 66.4 & 0.0 \\
\hline México & 171.0 & 36.7 & 63.3 & 0.0 \\
\hline Michoacán & 425.3 & 9.8 & 20.7 & 69.4 \\
\hline Morelos & 15.2 & 0.0 & 100.0 & 0.0 \\
\hline Nayarit & 99.6 & 15.3 & 18.4 & 66.3 \\
\hline Nuevo León & 12.1 & 0.0 & 100.0 & 0.0 \\
\hline Oaxaca & 746.6 & 17.5 & 16.6 & 65.9 \\
\hline Puebla & 563.4 & 23.2 & 18.6 & 58.2 \\
\hline Querétaro & 59.6 & 45.1 & 54.9 & 0.0 \\
\hline Quintana Roo & 16.7 & 0.0 & 100.0 & 0.0 \\
\hline San Luis Potosí & 362.9 & 13.1 & 20.0 & 66.9 \\
\hline Sinaloa & 202.4 & 15.2 & 18.3 & 66.5 \\
\hline Sonora & 19.9 & 0.0 & 100.0 & 0.0 \\
\hline Tabasco & 64.4 & 0.0 & 100.0 & 0.0 \\
\hline Tamaulipas & 121.9 & 0.0 & 18.0 & 82.0 \\
\hline Tlaxcala & 12.4 & 0.0 & 100.0 & 0.0 \\
\hline Veracruz & 744.9 & 18.0 & 22.2 & 59.8 \\
\hline Yucatán & 239.1 & 13.8 & 17.0 & 69.2 \\
\hline Zacatecas & 279.5 & 10.2 & 17.1 & 72.7 \\
\hline
\end{tabular}

Progresa: Programa de Educación, Salud y Alimentación

Fuente: Secretaría de Salud. Dirección General de Información y Evaluación del Desempeño. Boletín de Información Estadística No. 2I, 200I,Volumen IV 


\section{Cuadro IX \\ Gasto público en salud per capita por condición de aseguramiento. México, 200 I (PESOS CORRIENTES)}

Entidad federativa

Nacional

Aguascalientes

Baja California

Baja California Sur

Campeche

Coahuila

Colima

Chiapas

Chihuahua

Distrito Federal

Durango

Guanajuato

Guerrero

Hidalgo

Jalisco

México

Michoacán

Morelos

Nayarit

Nuevo León

Oaxaca

Puebla

Querétaro

Quintana Roo

San Luis Potosí

Sinaloa

Sonora

Tabasco

Tamaulipas

Tlaxcala

Veracruz

Yucatán

Zacatecas
Población asegurada

1.988

1.735

1.794

2.828

2.260

1.891

2.040

I.37I

I.677

4.943

$\mathrm{I} .607$

1.546

I.6II

1.507

1.763

959

1.499

1.885

2.023

1.911

1.088

1.632

1.479

$\mathrm{I} .879$

I 578

I 532

1795

2.153

1.906

1.508

2.031

1.969

1.524
Población no asegurada

Población total

1.045

1.527

$\begin{array}{ll}1.789 & 1.754\end{array}$

$980 \quad 1.537$

$2.754+2.806$

$\begin{array}{ll}1.718 & 1.994\end{array}$

$\begin{array}{ll}1.911 & 1.895\end{array}$

$1.508 \quad 1.822$

$748 \quad 891$

$1.209 \quad 1.530$

2.425

$\begin{array}{ll}1.413 & 1.529\end{array}$

$\begin{array}{ll}648 & 1.036\end{array}$

$817 \quad 1.043$

$992 \quad 1.180$

$\begin{array}{ll}1.136 & 1.488\end{array}$

905

$\begin{array}{ll}629 & 916\end{array}$

$887 \quad 1.334$

$\begin{array}{ll}1.156 & 1.570\end{array}$

$\begin{array}{ll}1.279 & 1.769\end{array}$

$837 \quad 910$

$\begin{array}{ll}574 & 932\end{array}$

$\begin{array}{ll}1.105 & 1.316\end{array}$

$\begin{array}{ll}1.607 & 1.770\end{array}$

$868+1.192$

$1.393 \quad 1.484$

$\begin{array}{ll}1.859 & 1.814\end{array}$

$\begin{array}{ll}1.859 & 1.837\end{array}$

$\begin{array}{ll}1.427 & 1.736\end{array}$

$843 \quad 1.116$

$\begin{array}{ll}774 & 1.283\end{array}$

1.329

889

Notas

I. El presupuesto total para población no asegurada incluye: Ramo 12, Ramo 33 y Aportaciones Estatales. Y dentro del Ramo I2 incluye PAC, Progresa, IMSS-Solidaridad, Unidades Centrales, Organismos Descentralizados y Organos Desconcentrados. La distribución del Ramo 12 por entidad federativa se realizó de la siguiente manera: los programas de combate a la pobreza (PAC, Progresa e IMSS-Solidaridad) son transferencia directa a entidades federativas. Lo correspondiente a Unidades Centrales se distribuyó en función de la participación que cada entidad tiene en la población total nacional. El Hospital Manuel Gea González y el Hospital General de México se distribuye en función del lugar de procedencia de las consultas de primera vez. Los Institutos Nacionales, en función de los egresos hospitalarios. La Administración del Patrimonio de la Beneficencia Pública y el Hospital juárez de México, en función de la población no asegurada.Y finalmente, el resto de Organismos Descentralizados y Organos Desconcentrados, con base en la participación de cada entidad en el total de población

2. El presupuesto total para población asegurada incluye IMSS, ISSSTE y Pemex

3. Las poblaciones están contabilizadas a final de periodo por la Dirección General de Información y Evaluación del Desempeño (DGIED)

PAC:Programa de Ampliación de Cobertura

Progresa: Programa de Educación, Salud y Alimentación

IMSS: Instituto Mexicano del Seguro Social

ISSSTE: Instituto de Seguridad y Servicios Sociales de los Trabajadores del Estado

Pemex: Petróleos Mexicanos

Fuente: Secretaría de Salud. Dirección General de Información y Evaluación del Desempeño. Boletín de Información Estadística No. 2I, 200I,Volumen IV y Dirección General de Equidad y Desarrollo en Salud 
Cuadro $\mathbf{X}$

Gasto en salud como porcentaje del gasto público. México, 200 I

\begin{tabular}{|c|c|c|c|}
\hline Entidad federativa & Total & Población asegurada & Población no asegurada \\
\hline Nacional & 16.9 & 11.2 & 5.7 \\
\hline Aguascalientes & 18.6 & 12.0 & 6.6 \\
\hline Baja California & 18.5 & 14.8 & 3.7 \\
\hline Baja California Sur & 19.8 & 14.0 & 5.7 \\
\hline Campeche & 6.9 & 4.0 & 2.9 \\
\hline Coahuila & 22.8 & 18.1 & 4.6 \\
\hline Colima & 11.0 & 7.3 & 3.7 \\
\hline Chiapas & 13.0 & 4.6 & 8.4 \\
\hline Chihuahua & 20.6 & 15.5 & 5.1 \\
\hline Distrito Federal & 14.6 & 11.6 & 3.0 \\
\hline Durango & 5.1 & 3.2 & 1.9 \\
\hline Guanajuato & 21.4 & 13.8 & 7.6 \\
\hline Guerrero & 13.8 & 6.1 & 7.8 \\
\hline Hidalgo & 13.0 & 6.0 & 6.9 \\
\hline Jalisco & 31.1 & 20.6 & 10.4 \\
\hline México & 25.0 & 13.9 & 11.1 \\
\hline Michoacán & 18.7 & 10.1 & 8.6 \\
\hline Morelos & 22.0 & 13.9 & 8.1 \\
\hline Nayarit & 20.8 & 12.8 & 8.0 \\
\hline Nuevo León & 23.2 & 19.4 & 3.8 \\
\hline Oaxaca & 13.9 & 4.8 & 9.1 \\
\hline Puebla & 20.3 & 12.0 & 8.3 \\
\hline Querétaro & 16.2 & 10.3 & 5.9 \\
\hline Quintana Roo & 23.9 & 15.3 & 8.7 \\
\hline San Luis Potosí & 18.7 & 11.3 & 7.4 \\
\hline Sinaloa & 18.4 & 12.4 & 6.0 \\
\hline Sonora & 20.2 & 14.0 & 6.2 \\
\hline Tabasco & 15.8 & 7.3 & 8.5 \\
\hline Tamaulipas & 16.3 & 11.5 & 4.8 \\
\hline Tlaxcala & 20.4 & 11.3 & 9.1 \\
\hline Veracruz & 14.9 & 9.6 & 5.3 \\
\hline Yucatán & 19.1 & 12.6 & 6.4 \\
\hline Zacatecas & 16.4 & 8.5 & 7.9 \\
\hline
\end{tabular}

Notas

I. El presupuesto total para población no asegurada incluye: Ramo 12, Ramo 33 y Aportaciones Estatales. Y dentro del Ramo 12 incluye PAC, Progresa, IMSS-Solidaridad, Unidades Centrales, Organismos Descentralizados y Organos Desconcentrados. La distribución del Ramo I2 por entidad federativa se realizó de la siguiente manera: los programas de combate a la pobreza (PAC, Progresa e IMSS-Solidaridad) son transferencia directa a entidades federativas. Lo correspondiente a Unidades Centrales se distribuyó en función de la participación que cada entidad tiene en la población total nacional. El Hospital Manuel Gea González y el Hospital General de México se distribuye en función del lugar de procedencia de las consultas de primera vez. Los Institutos Nacionales, en función de los egresos hospitalarios. La Administración del Patrimonio de la Beneficencia Pública y el Hospital Juárez de México, en función de la población no asegurada.Y finalmente, el resto de Organismos Descentralizados y Organos Desconcentrados, con base en la participación de cada entidad en el total de población

2. El presupuesto total para población asegurada incluye IMSS, ISSSTE y Pemex

3. El gasto público equivale al gasto programable en la clasificación funcional

PAC:Programa de Ampliación de Cobertura

Progresa: Programa de Educación, Salud y Alimentación

IMSS: Instituto Mexicano del Seguro Social

ISSSTE: Instituto de Seguridad y Servicios Sociales de los Trabajadores del Estado

Pemex: Petróleos Mexicanos

Fuentes: Secretaría de Salud. Dirección General de Información y Evaluación del Desempeño. Boletín de Información Estadística No 2I, 200I Volumen IV, Cuenta de la Hacienda Pública y Dirección General de Equidad y Desarrollo en Salud 


\section{Cuadro XI \\ Gasto en salud como porcentaje del PiB. México, 200 I}

Entidad federativa

Nacional

Aguascalientes

Baja California

Baja California Sur

Campeche

Coahuila

Colima

Chiapas

Chihuahua

Distrito Federal

Durango

Guanajuato

Guerrero

Hidalgo

Jalisco

México

Michoacán

Morelos

Nayarit

Nuevo León

Oaxaca

Puebla

Querétaro

Quintana Roo

San Luis Potosí

Sinaloa

Sonora

Tabasco

Tamaulipas

Tlaxcala

Veracruz

Yucatán

Zacatecas
Total

2.7

\section{7}

2.2

4.0

2.3

2.9

3.6

4.2

2. 1

3.0

3.5

3.1

3.6

4.0

2.8

2.4

3.4

2.8

5.0

5.0
1.9

4. 1

2.4

2.1

2.0

3.3

3.9

2.9

5.5

3.1

3.8

4.4

3.8

4.2

Población asegurada

1.8

$\begin{array}{ll}1.7 & 1.0\end{array}$

1.70 .4

$\begin{array}{ll}1.9 & 1.2 \\ 1.3 & 1.0\end{array}$

$\begin{array}{ll}1.3 & 1.0\end{array}$

$\begin{array}{ll}2.3 & 0.6 \\ 2.4 & 1.2\end{array}$

$\begin{array}{ll}1.5 & 2.7\end{array}$

1.60 .5

$\begin{array}{ll}1.6 & 0.5 \\ 2.4 & 0.6\end{array}$

$\begin{array}{ll}2.4 & 0.6 \\ 2.0 & 1.3\end{array}$

$\begin{array}{ll}2.2 & 1.3 \\ 2.0 & 1.1\end{array}$

$\begin{array}{ll}1.6 & 2.0\end{array}$

1.9202 .1

$\begin{array}{ll}1.9 & 1.0\end{array}$

$\begin{array}{ll}1.3 & 1.1\end{array}$

$\begin{array}{ll}1.8 & 1.5\end{array}$

$\begin{array}{ll}1.8 & 1.0\end{array}$

$\begin{array}{ll}1.1 & 1.9\end{array}$

$\begin{array}{ll}3.1 & 1.9 \\ 1.6 & 0.3\end{array}$

$\begin{array}{ll}1.6 & 0.3 \\ 1.4 & 2.7\end{array}$

$\begin{array}{ll}1.4 & 2.7 \\ 1.4 & 1.0\end{array}$

$\begin{array}{ll}1.4 & 0.8\end{array}$

$\begin{array}{ll}1.3 & 0.7\end{array}$

$\begin{array}{ll}2.0 & 1.3\end{array}$

$\begin{array}{ll}2.6 & 1.3\end{array}$

$\begin{array}{ll}2.0 & 0.9\end{array}$

$2.6 \quad 3.0$

$\begin{array}{ll}2.6 & 3.0 \\ 2.2 & 0.9\end{array}$

$2.1 \quad 1.7$

$2.8 \quad 1.7$

$\begin{array}{ll}2.8 & 1.6 \\ 2.5 & 1.3 \\ 2.2 & 2.0\end{array}$

Notas

I. El presupuesto total para población no asegurada incluye: Ramo 12, Ramo 33 y Aportaciones Estatales. Y dentro del Ramo 12 incluye PAC, Progresa, IMSS-Solidaridad, Unidades Centrales, Organismos Descentralizados y Organos Desconcentrados. La distribución del Ramo 12 por entidad federativa se realizó de la siguiente manera: los programas de combate a la pobreza (PAC, Progresa e IMSS-Solidaridad) son transferencia directa a entidades federativas. Lo correspondiente a Unidades Centrales se distribuyó en función de la participación que cada entidad tiene en la población total nacional. El Hospital Manuel Gea González y el Hospital General de México se distribuye en función del lugar de procedencia de las consultas de primera vez. Los Institutos Nacionales, en función de los egresos hospitalarios. La Administración del Patrimonio de la Beneficencia Pública y el Hospital Juárez de México, en función de la población no asegurada.Y finalmente, el resto de Organismos Descentralizados y Organos Desconcentrados, con base en la participación de cada entidad en el total de población

2. El presupuesto total para población asegurada incluye IMSS, ISSSTE y Pemex

PAC:Programa de Ampliación de Cobertura

Progresa: Programa de Educación, Salud y Alimentación

IMSS: Instituto Mexicano del Seguro Social

ISSSTE: Instituto de Seguridad y Servicios Sociales de los Trabajadores del Estado

Pemex: Petróleos Mexicanos

Fuentes: Secretaría de Salud. Dirección General de Información y Evaluación del Desempeño. Boletín de Información Estadística No. 2I, 200 I Volumen IV, Cuenta de la Hacienda Pública y Dirección General de Equidad y Desarrollo en Salud 


\section{Notas metodológicas}

\section{Distribución del Ramo 12}

La distribución del ramo 12 se realizó de la manera siguiente:

1. Para Unidades Centrales, la distribución fue hecha en función de la población total.

2. En cuanto a los Organos Desconcentrados, se hace lo siguiente: lo correspondiente a la Administración del Patrimonio de la Beneficencia Pública y el Hospital Juárez de México fue distribuido en función de la población no asegurada. Para el resto de los órganos se distribuye en función de la población total (asegurada y no asegurada).

3. Para la distribución de Organismos Descentralizados se procedió de la siguiente forma: los Institutos Nacionales (Psiquiatría, Cancerología, Cardiología, Enfermedades Respiratorias, Nutrición, Senectud, Neurología y Neurocirugía, Pediatría y Perinatología) más el Hospital Infantil de México fue distribuido en función de egresos hospitalarios. IMSS-Sol ya viene distribuido en el Boletín de Información Estadística (BIE), por lo que se toma como se reporta por entidad. El Hospital Manuel Gea González y el Hospital General de México está distribuido con base en el lugar de procedencia de las consultas de primera vez. Para el resto de los organismos se distribuye en función de la población total (asegurada y no asegurada).

3. En cuanto a los programas de ramo 12 (PAC, Progresa e IMSS-Solidaridad) ya vienen desagregados a nivel estatal en el Boletín de Información Estadística 2001, por ello se tomó la cifra que reporta cada entidad en estos programas.
Poblaciones utilizadas en el análisis:

$\begin{array}{lccc}\text { Entidad federativa } & \begin{array}{c}\text { Población } \\ \text { total }\end{array} & \begin{array}{c}\text { Población } \\ \text { no asegurada }\end{array} & \begin{array}{c}\text { Población } \\ \text { asegurada }\end{array} \\ \text { Nacional } & 102222347 & 50014195 & 52208152\end{array}$

\begin{tabular}{|c|c|c|c|}
\hline Aguascalientes & 999816 & 348498 & 651318 \\
\hline Baja California & 2602077 & $822 \quad 143$ & 1779933 \\
\hline Baja California Sur & 443316 & 130686 & $31263 \mid$ \\
\hline Campeche & 729779 & $358 \quad 172$ & $37 \mid 606$ \\
\hline Coahuila & 2426888 & 490375 & 1936514 \\
\hline Colima & 564294 & 231233 & 333061 \\
\hline Chiapas & 4158722 & 3205580 & $953 \quad 44$ \\
\hline Chihuahua & 3195963 & $|00282|$ & $2|93| 4 \mid$ \\
\hline Distrito Federal & 8822936 & 3033128 & 5789808 \\
\hline Durango & $|54| 198$ & 623674 & 917525 \\
\hline Guanajuato & 4976544 & $2827 \quad 135$ & 2149408 \\
\hline Guerrero & 3234194 & 2315669 & 918525 \\
\hline Hidalgo & 2345505 & I 489835 & 855670 \\
\hline Jalisco & 6625653 & 2913092 & 3712562 \\
\hline México & 13699944 & 6282268 & 7417677 \\
\hline Michoacán & 4275554 & 2865611 & I 409943 \\
\hline Morelos & 1641918 & 906767 & $735 \quad 152$ \\
\hline Nayarit & 974205 & 508855 & 465351 \\
\hline Nuevo León & 3999231 & 898139 & 3101092 \\
\hline Oaxaca & 3639310 & 2590990 & 1048319 \\
\hline Puebla & $52864 \mid 4$ & 3495311 & | 79| 103 \\
\hline Querétaro & I 484816 & 644929 & 839886 \\
\hline Quintana Roo & 912611 & 364272 & 548339 \\
\hline San Luis Potosí & 2421463 & 1314608 & I 106855 \\
\hline Sinaloa & $2624|2|$ & 909385 & 1714736 \\
\hline Sonora & 2332246 & 699173 & 1633073 \\
\hline Tabasco & 1985439 & I 200055 & 785384 \\
\hline Tamaulipas & 2900764 & 1030236 & I 870528 \\
\hline Tlaxcala & 1015380 & 599006 & 416374 \\
\hline Veracruz & 7193931 & 4280701 & 2913230 \\
\hline Yucatán & 1719903 & 740042 & 979860 \\
\hline Zacatecas & | 448214 & 891807 & 556407 \\
\hline
\end{tabular}

Nota

a) Las poblaciones están contabilizadas a final de periodo por la Dirección General de Información y Evaluación del Desempeño (DGIED)

\section{Acrónimos}

FASSA Fondo de Aportaciones para los Servicios de Salud IMSS Instituto Mexicano del Seguro Social

ISSSTE Instituto de Seguridad y Servicios Sociales de los Trabajadores del Estado
PAC Programa de Ampliación de Cobertura

Pemex Petróleos Mexicanos

Progresa Programa de Educación, Salud y Alimentación 


\section{Glosario de términos}

Aportaciones estatales. Recursos canalizados por el gobierno del Estado para el financiamiento de la salud.

Capítulo de gasto. Son elementos de la clasificación por objeto del gasto que constituyen un conjunto homogéneo, claro y ordenado de los bienes y servicios que el Gobierno Federal y las entidades paraestatales adquieren para la consecución de sus objetivos y metas. La unidad básica de registro que conforma un capítulo presupuestario es la "partida"; un conjunto de partidas forman un "concepto" y un grupo de conceptos integran un "capítulo". Este nivel de agregación hace posible el análisis retrospectivo y prospectivo de los planes o programas de acuerdo con la naturaleza del gasto a realizar. Los capítulos que se emplean para el sector salud son:

1000 servicios personales

2000 materiales y suministros

3000 servicios generales

4000 transferencias

5000 bienes muebles e inmuebles

6000 obras públicas

7000 inversión financiera

Consulta de primera vez. La primera atención que otorga el médico general o familiar a los pacientes ambulatorios en la unidad médica o en el domicilio, con objeto de proporcionar asistencia médica o procedimientos preventivos.

Egresos hospitalarios. Es el paciente que sale de un servicio del hospital, por diversos motivos: altas por curación, mejoría, traslado a otra unidad, defunción, alta voluntaria o fuga, e implica la desocupación de una cama censable.

Fondos. Los recursos destinados a la operación promoción y restauración de la salud; los canalizan a las instituciones prestadoras de servicios.

Fuentes. Entidades emisoras de los recursos destinados a promover o restablecer la salud de la población.

Gasto en salud. Mide el uso final de las unidades residentes de bienes y servicios de atención de la salud; más la formación bruta de capital de las ramas de actividad proveedoras de atención de la salud (instituciones cuya actividad principal es la atención de la salud). Incluye por lo tanto el gasto en rectoría (diseño e implantación de políticas públicas, información y evaluación y regulación y normatividad), la prestación de servicios colectivos de salud, la prestación de servicios de salud a la persona, la producción de bienes para la salud y la investigación científica, y desarrollo tecnológico y de recursos humanos en el ámbito de la salud.

Gasto programable. Es el que se destina a las instituciones del Gobierno Federal para que éstas puedan proporcionar ser- vicios. Dicho gasto se canaliza a los Poderes e Instituto Federal Electoral (IFE), entidades paraestatales sujetas a control presupuestal y a las dependencias y ramos del Ejecutivo Federal.

IMSS-Solidaridad. Programa que opera con fondos del Gobierno Federal, se apoya en la estructura administrativa del Instituto Mexicano del Seguro Social (IMSS) y funciona en forma desconcentrada. Tiene como objetivo fundamental proporcionar servicios de salud oportunos y de calidad a población campesina e indígena de su ámbito de responsabilidad, a través de su modelo de Atención Integral a la Salud, que se sustenta fundamentalmente en la participación comunitaria.

Indicadores de resultados. Expresiones objetivas que permiten, a través de la relación cuantitativa entre dos o más variables que intervienen en un mismo proceso, dar seguimiento a los objetivos intermedios de los sistemas estatales y nacionales de salud, los mismos que a su vez se identifican con los atributos deseables de estos sistemas: anticipación, accesibilidad, aceptabilidad, calidad, cobertura, efectividad (resultados en salud), eficiencia, equidad, salud financiera y sensibilidad de género. Dichos indicadores fueron aprobados por el Consejo Nacional de Salud (CNS).

Organismo descentralizado. Institución definida por la Ley Orgánica de la Administración Pública Federal con personalidad jurídica y patrimonio propio, constituida con fondos o bienes provenientes de la Administración Pública Federal; su objetivo es la prestación de un servicio público o social, la explotación de bienes o recursos propiedad de la nación, la investigación científica y tecnológica y la obtención y aplicación de recursos para fines de asistencia o seguridad social.

Organo desconcentrado. Institución con autonomía administrativa, pero sin personalidad jurídica ni patrimonio propio, que de acuerdo con la Ley Orgánica de la Administración Pública Federal tiene facultades específicas para resolver asuntos de la competencia de su órgano central, siempre y cuando siga los señalamientos de normatividad dictados por este último.

Per capita. Por habitante.

Población asegurada. Segmento de la población total, que mediante una contribución o un pago anticipado tiene derecho a utilizar los servicios de seguridad social.

Población no asegurada. Son todas aquellas personas que no tienen acceso al sistema de seguridad social.

Población total. Comprende a la población del país, conformada por edad, sexo y entidad federativa.

Precios constantes. Indicador que expresa el valor de las mercancías y servicios a precio de un año base. 
Precios corrientes. Indicador del valor de las mercancías o servicios acumulados al momento de la operación; se emplea para referirse a los valores de las mercancías, expresados a precios de cada año.

Presupuesto ejercido. Importe de las erogaciones realizadas respaldado por los documentos comprobatorios (facturas, notas, nóminas, etcétera) presentados a la dependencia o entidad una vez autorizadas para su pago, con cargo al presupuesto autorizado.

Programa. Conjunto homogéneo y organizado de actividades a realizar para alcanzar una o varias metas, con recursos previamente determinados y a cargo de una unidad responsable.

Ramo administrativo. Es cada uno de los organismos, dependencias o instituciones por medio de los cuales se identifican los recursos que se les asignan en el Presupuesto de
Egresos de la Federación, se incluyen los Poderes Legislativo y Judicial de la Federación.

Ramo 12. Es el ramo administrativo destinado al sector salud. Ramo 33. Aportaciones federales para entidades federativas y municipios. Incluye varios fondos de los cuales el Fondo de Aportaciones para los Servicios de Salud (FASSA) se destina al financiamiento de servicios de salud en las entidades federativas.

Rubro. Título que se utiliza para agrupar un conjunto de cuentas.

Unidades centrales. Forman parte de la Secretaría de Salud, tienen la función de rectoría, es decir, que tienen la facultad y capacidad del Estado para dirigir la actividad de los agentes económicos al logro de los objetivos y metas del desarrollo. 